\title{
Dynamical stripe correlations and the spin fluctuations in cuprate superconductors
}

\author{
J. Zaanen and W. van Saarloos ${ }^{\mathrm{a}}$ \\ ${ }^{a}$ Institute Lorentz for Theoretical Physics, Leiden University, \\ P.O. Box 9506, NL-2300 Leiden, The Netherlands
}

It is conjectured that the anomalous spin dynamics observed in the normal state of cuprate superconductors might find its origin in a nearly ordered spin system which is kept in motion by thermally meandering charged domain walls. 'Temperature sets the scale' finds a natural explanation, while a crossover to a low temperature quantum domain wall fluid is implied.

\section{Introduction}

Much excitement is generated by the observation by Tranquada et al. of the striped phase in the cuprates[1], which appears to be in a tight competition with the superconducting state as long as the doping level is not too high[2]. Taken together with the observation of the persistent gap in the normal state[3] and the finding that a large magnetic field stabilizes an insulating state[4], this points to a bosonic physics. The electrons pair up above $T_{c}$, forming an interacting boson system and as in, e.g., ${ }^{4} \mathrm{He}$ the crystalline ('charge-ordered') and superconducting state appear as the natural candidates for the ground state.

The real novelty of the striped phase is that it is at the same time a Néel spin condensate. At least, it is a bound state of charge and spin[5], with the specialty that the charge sector couples into the spin sector in the form of disorder operators: the charged stripes are anti-phase boundaries in the Néel state. In fact, all evidence for persisting dynamical stripe correlations in the superconductors rests entirely on the spin sector[2].

Initially we considered the possibility of stripelike correlations, motivated by the anomalous behavior of the spin-fluctuations in the cuprates[6]. Both inelastic neutron scattering $[8,9]$ and NMR[7] reveal that these fluctuations show a characteristic time- $(\tau)$ and length-scale $(\xi)$ which are both precisely inversely proportional to temperature. The lack of an intrinsic scale is rem- inescent of a (quantum) critical regime. This is the underlying idea behind the 'nearly antiferromagnetic Fermi liquid' idea of Pines and coworkers[10]: the system is on its way to a zero temperature ordered spin state, and superconductivity intervenes at the last moment.

There is a conceptual difficulty with this scenario. It is expected that temperature and doping play the role of control parameters: how can it be that the critical regime extends over a temperature- and doping range of $\sim 1000 \mathrm{~K}$ and $\sim 10 \%$, respectively? At least classical critical regimes do not behave in this way. Assuming that the system is on its way to the striped phase, the relative insensitivity to doping dependence is trivially explained. In contrast to normal antiferromagnets, the striped phase is an incommensurate solid which can exist over a large doping range. The non-trivial part is to explain the temperature dependences of the characteristic dynamical scales.

The problem of thermally fluctuating domain walls in two dimensions ('incommensurate fluids') has been studied in great detail[11]. In such systems, the phenomenon of entropic repulsions is common. For increasing temperature, the thermal meandering motions of individual domain walls increases, as well as the number of collisions between domain walls. Collisions cost entropy and the net effect is that the stiffness of the system as a whole increases. Due to these entropic repulsion effects, temperature becomes an impor- 
tant parameter in the dynamics. It is emphasized that the basic effect is unrelated to criticality and it can therefore appear over a large temperature range. As we showed in an earlier paper[6], the behaviors of the characteristic time- and length scales of the spin fluctuations match in a natural way with the expectations for a thermally fluctuating incommensurate fluid in two dimensions.

Although the essence of our earlier work is unchanged, the relationship between the domain wall dynamics and the observed spin dynamics was treated too casually in this earlier paper[6]. After summarizing the basics, we will make this connection more precise. This is used, together with a refined experimental characterization of the normal state magnetic fluctuations[9], to deduce a more complete picture of the domain wall fluid.

\section{Incommensurate fluids in two dimen- sions.}

The standard theory of incommensurate fluids[11] starts with the assumption that single domain walls (DW) behave like Gaussian strings. It is assumed that its motions can be completely parametrized in terms of a transversal sound mode $\omega_{q}=c_{W} q$, where $q$ is the wave number along the string, while $c_{W}=\sqrt{\Sigma / \rho}$ ( $\Sigma$ is the string tension and $\rho$ the mass density). In addition, it is convenient to first only consider hard core string-string interactions. At finite temperatures, DW will be subject to meandering motions. The mean square fluctuation of a single string in $2 \mathrm{D}$ in the transverse $z$ direction between two points separated by a distance $l$ diverges as $\left\langle\left(\Delta z_{l}\right)^{2}\right\rangle \simeq \frac{k_{B} T}{\Sigma} l$. When the walls have an average distance $d$, they will on average collide when $\left\langle\left(\Delta z_{l_{\mathrm{c}}}\right)^{2}\right\rangle \simeq d^{2}$. The typical distance between collision points follows immediately,

$l_{c} \simeq \frac{\Sigma d^{2}}{k_{B} T}$

This is the most important length scale in this problem. It sets (a) the crossover length where the system changes from single wall dynamics to that of the domain wall fluid, (b) it determines the collision density and thereby the strength of the entropic repulsions, and (c) it corresponds to the shortest spin-spin disordering length in the special case of the striped fluid. For a further elaboration of the statistical physics we refer to the literature[11]. The essence is that the domain wall system can be described as a 2D elastic medium with an elastic constant which itself is proportional to temperature: $F \sim T \int d \vec{r}(\Delta \vec{n})^{2}$. This is like a XY spin system with $J \sim T$, and it follows that it is characterized by a zero temperature Kosterlitz-Thouless transition: at every finite temperature, free dislocations proliferate and the system is a fluid.

In order to adress the temporal aspects of the dynamics we suggested that the single wall dynamics is coherent on length scales $\leq l_{c}$ : the single string sound oscillations are assumed to be underdamped. If this is the case, the relative motion of two points a distance $l$ apart becomes important after a time $l / c_{W}$, and likewise the mean average fluctuations of two points a distance $l_{c}$ apart deviates significantly from the value $d$ after a time of order $l_{c} / c_{W}$. Using Eq. (1), it follows that the collision frequency is of order[6],

$\hbar \Gamma=\mu k_{B} T, \mu=\frac{\pi \hbar}{\rho c_{W} d^{2}}$

Hence, we find both a characteristic length- and time scale which vary as $1 / T$, with the obviously important meaning that they both refer to the crossover from single wall to many wall behavior. It remains to be demonstrated that these characteristic scales govern the spin dynamics as well.

\section{Charged domain walls and spin fluctua- tions.}

The unique feature of the stripes is that it corresponds with an incommensurate solid/fluid problem in the charge/longitudinal sector, coupled into a spin problem: when the stripes are ordered one is still left with a Heisenberg antiferromagnet. Besides the modes of the previous section, one has to deal with the transversal fluctuations associated with the rotational invariance of the spin system. At least on the classical level, the domain wall- and the tranversal spin fluctuations appear to decouple in the infrared. Although 
this is theoretically not well understood, this appears to be implied by the fact that the chargeand spin ordering transitions are decoupled[1,12]. This can be stated more precisely as follows: as long as the charged domain walls are intact, the spin system is unfrustrated. Calling the exchange interaction inside the spin domains $J$ and that between spins on opposite sides of the wall $J^{\prime}$, the (classical) spin system appears identical for every possible configuration of domain walls when $J=J^{\prime}$. Apparently, $J-J^{\prime}$ is an irrelevant operator. Hence, although microscopically $J \neq J^{\prime}$ (and frustration), the spin- and domain wall dynamics decouple in the approach to the long wave length limit, although the numerical factors (like the spin wave velocity) have to be adjusted.

Does this mean that the domain wall dynamics would be completely invisible in a measurement of the spin dynamical form factor? In fact, in this way one measures a convolution of (transversal) spin- and domain wall dynamics. One can define two distinct equal time correlators $\left(\vec{M}_{i}=(-1)^{i} \vec{S}_{i}\right.$ is staggered spin),

$$
\begin{aligned}
& S(R)=\left\langle\vec{M}_{i+R} \cdot \vec{M}_{i}\right\rangle \sim e^{-R / \xi} \\
& S_{T}(R)=\left\langle\vec{M}_{i+R}\left[\Pi_{l=i+R}^{i} e^{i \pi \hat{n}_{l}}\right] \vec{M}_{i}\right\rangle \sim e^{-R / \xi_{T}}
\end{aligned}
$$

where the last proportionalities hold when the spin system is disordered. In Eq. (4), $\hat{n}$ measures the charge associated with a single domain wall unit cell (e.g., in the nickelates $\hat{n}$ corresponds with the hole number operator, while in the halffilled cuprate walls $\hat{n}=1$ corresponds with half a hole). Hence, the charge sector appears in a disorder operator form in the spin correlator Eq. (4): regardless of the location of the domain walls, the change of sign of the spin order parameter upon crossing the walls is undone by the term between square brackets. It is this correlator which measures the transversal spin correlations. On the other hand, in the experiment one measures the correlator Eq.(3), which is also sensitive for the disorder induced by the domain wall fluid. This general wisdom should also apply to unequal time correlations.

With the above observations, it becomes possible to deduce some characteristics of the $\vec{q}$ and $\omega$ dependence of the spin dynamical structure factor. Let us consider the case that Eq. (4) would show near-long-range order, while the charge sector is an incommensurate fluid $\left.\left(\xi_{T}>\right\rangle\right)$. To address the temporal aspects of the dynamics it is convenient to consider the imaginary part of the local dynamical spin susceptibility $\chi^{\prime \prime}(\omega)=$ $\int d \vec{q} \chi^{\prime \prime}(\vec{q}, \omega)$ : every time a domain wall passes a particular site, the spin at this site is flipped and $\chi^{\prime \prime}(\omega)$ is dominated by this dissipation source. It is easy to see that the domain wall scale $\Gamma$, Eq. (2), should appear as a characteristic scale in $\chi^{\prime \prime}$ : (a) when $\omega>>\Gamma$ the domain wall system appears as static and the frequency dependence should be similar to that of the pure spin system, (b) when $\omega \rightarrow 0$ the spin-flip rate is nonzero as long as the domain walls are in a fluid state (even when $\xi_{T} \rightarrow \infty$ ) and on hydrodynamic grounds $\chi^{\prime \prime}(\omega) \sim \omega$. In ref.[6] we present a more detailed hydrodynamic argument showing that in fact $\chi^{\prime \prime}(\omega, T) \sim \omega / T$. The crossover regime between the high- and low frequency regimes should be governed by the single scale parameter $\Gamma$, and $\chi^{\prime \prime}(\omega, T) \sim F(\omega / \Gamma)=F\left(\omega /\left(\mu k_{B} T\right)\right.$, and this is the scaling observed in neutron scattering [8]. Hence, we suggest that this crossover regime is actually probed in the inelastic neutron scattering experiments. As an interesting ramification, the prefactor $\mu$ in Eq. (2) is also the dimensionless quantum of action of the incommensurate fluid: the characteristic energy scale for quantum fluctuations $\hbar \Gamma_{Q}=\left(\hbar c_{W} / a\right) \exp (-1 / \mu)[6]$. If, and only if, $\mu$ is of order unity a crossover is expected at a reasonable temperature from a high temperature classical fluid to a low temperature quantum regime (like in ${ }^{4} \mathrm{He}$ ) and this appears as a consistency requirement in the present context. Experimentally, $\mu \simeq 1$ [8].

A little more can be said regarding the spatiotemporal appearance of the magnetic fluctuations. Assuming the near decoupling of the transversal- and domain wall dynamics, the spin wave (transversal mode) spectrum of the static striped phase appears as a sensible zeroth order: the goldstone modes attach to the incommensurate wave vectors and the branches should come together at the $(\pi, \pi)$ point, while gaps $\sim J-J^{\prime}$ are found at the new Brillioun zone boundaries at 
smaller momenta[13]. When the domain walls are disordered, one expects that the spin waves start to propagate when their wave length becomes of order of, or less than the disordering length of the domain wall fluid. Longer wavelength spin waves will be overdamped. Hence, as a function of frequency a crossover has to occur from an overdamped response at low frequencies to an underdamped spin wave response at higher frequencies. The frequency where this occurs is $\omega_{c} \sim c \pi / l_{m . f}$., where the mean-free path of the spin waves is expected to be proportional to the domain wall disordering length: $l_{m . f .} \simeq \alpha \xi_{c}$, where $\alpha$ is a spin wave-domain wall scattering cross section $(0<\alpha<1)$. At frequencies much less than $\omega_{c}$, the width in momentum space should be set by $\pi / l_{m . f .}$. As long as the spin-wave cones are unresolved, the width of the peaks in $S(\vec{q}, \omega)$ should behave as a simple geometric average of the inverse domain wall disordering length $\left(1 /\left(\alpha \xi_{c}\right)\right)$ and the apparent $\omega$ dependent width coming from the unresolved spin-wave cone $\left(\kappa_{\omega}=\omega / c\right)$. The domain wall disordering length is nothing else than the collision length (Eq.1) and we expect for the frequency- and temperature dependence of the width of the incommensurate peaks,

$\kappa(\omega, T)=\sqrt{\frac{\left(k_{B} T\right)^{2}}{\left(\alpha \Sigma d^{2}\right)^{2}}+\frac{\omega^{2}}{c^{2}}}$

where $c$ is the spin-wave velocity.

Except for a residual width, this is the behavior found in the experiment[9]. Interestingly, Aeppli et al find that temperature and frequency appear with common prefactors. Together with $\mu \simeq 1$ this implies that $\alpha c_{W} \simeq c$ : the product of the spin wave-domain wall cross section and the wall transversal sound velocity is of order of the spin wave velocity $(c \simeq 0.2 \mathrm{eV} \AA$ ). This observation might hint at the microscopic mechanism of stripe formation.

Further experimentation is needed to see if the above physics is the correct one. Central to our analysis is the assertion that the collective dynamics in the normal state is in a classical regime. General arguments indicate that the difference between Eqs. (3,4) acquires a different meaning in the quantum system, and it is tempting to speculate that the coherent spin mode observed by Mason et al [14] reflect the onset of quantum coherence in the domain wall fluid upon entering the superconducting regime.

Aknowledgements. We acknowledge stimulating discussions with G. Aeppli, A. V. Balatski, D. Pines, M. Horbach, S. Kivelson, D. H. Lee and J. Tranquada. JZ acknowledges financial support by the Dutch Academy of Sciences (KNAW).

\section{REFERENCES}

1. J. M. Tranquada et al, Nature 375, 561 (1995).

2. J. M. Tranquada et al, Phys. Rev. Lett. 78, 338 (1997).

3. B. G. Levi, Physics Today 49 (6), 17 (1996).

4. G. S. Boebinger et al, Ground state of superconducting $\mathrm{La}_{2-x} \mathrm{Sr}_{x} \mathrm{CuO}_{4}$ in 61-Tesla magnetic fields, Bell Labs. preprint (1996).

5. J. Zaanen and O. Gunnarsson, Phys. Rev. B 39, 236 (1989).

6. J. Zaanen, M. L. Horbach and W. van Saarloos, Phys. Rev. B 53, 8671 (1996).

7. T. Imai et al, J. Phys. Soc. Jpn. 59, 3846 (1990).

8. T. E. Mason et al, Phys. Rev. Lett. 68, 1414 (1992).

9. G. Aeppli et al, Magnetic fluctuations in the normal state of the high $T_{c}$ superconductor $\mathrm{La}_{1.86} \mathrm{Sr}_{0.14} \mathrm{CuO}_{4}$, NEC preprint (1996).

10. P. Monthoux, A. V. Balatski and D. Pines, Phys. Rev. Lett. 67, 3448 (1991).

11. see S. N. Coppersmith et al, Phys. Rev. B 25, 349 (1982) and refs. therein.

12. J. M. Tranquada et al, Phys. Rev. Lett. 73, 1003 (1994); J. M. Tranquada et al, Chargedriven stripe order of holes and spins in $\mathrm{La}_{2-x} \mathrm{Sr}_{x} \mathrm{NiO}_{4}$ with $\mathrm{x}=0.225$, Phys. Rev. $B$ (in press).

13. J. M. Tranquada et al, spin dynamics in an ordered stripe phase, cond-mat/9612007.

14. T. E. Mason et al, Phys. Rev. lett. 77, 1604 (1996). 